\title{
PARTINGTON-ROCHELLE PANCREATICOJEJUNOSTOMY FOR CHRONIC PANCREATITIS COMBINED WITH CHOLANGIOENTEROSTOMY TREATMENT OF CHRONIC PANCREATITIS AND BILIARY STRICTURES: CASE REPORT AND REVIEW OF THE LITERATURE
}

\author{
Abdul Malik', Lu-Lu Liu², Ming-Ming Sun ${ }^{3}$, Ahamd Daniyal Shahid ${ }^{4}$, Song Zou ${ }^{5}$, Lei Cui' ${ }^{6}$, Ji-Xiang Chen ${ }^{7}$, \\ Abdul Raheem ${ }^{8}$, Min Gu', Sheng-Chun Dang*10, \\ *1.2.3.4.5.6.7.8.10 (Department of General Surgery, The Affiliated Hospital of Jiangsu University, Zhenjiang 212001, Jiangsu \\ Province, China) \\ ${ }^{9}$ (Zhenjiang Integrative Medicine Hospital, Zhenjiang 212001, Jiangsu Province, China
}

*Corresponding Author: -

Email: China.dscgu@163.com

\begin{abstract}
: -
Chronic pancreatitis (CP) is a kind of pancreatic disease with irreversible morphological changes, can lead to impaired endocrine and exocrine function of the inflammatory process. CP simultaneously accompanied with bile duct stricture $(B D S)$ is a rare condition. At present, the treatment of $C P$ tend to be individualized by the surgeons. Here, we reported the case of a plastic stent inserted two times into the main pancreatic duct and common bile duct. During the last 5 years, the patient hospitalized several times due to recurrent abdominal pain, till Partington-Rochelle pancreatojejunostomy (PRP) procedure combined with cholangioenterostomy was treated without complications. After 4 years of follow-up, the patient showed abnormal glucose metabolism without the need for additional enzymes, and he was in good condition without pain. Partington-Rochelle procedure combined with cholangioenterostomy provided a promising result of pancreatitis with BDS endoscopic papillary and stent failure.
\end{abstract}

Keywords: - chronic pancreatitis; bile duct stricture; Partington-Rochelle procedure; Puestow procedure.

\section{(c) $(\$)$}




\section{INTRODUCTION (11 BOLD)}

The introduction Chronic pancreatitis is a variety of pathogenic inflammatory disease. characterized by substantial parenchymal destruction of the pancreas and subsequent fibrosis ${ }^{1}$. Pain is the main symptom of most patients with chronic pancreatitis. The causes of pain is multifactorial, though the main risk factors of pain in chronic pancreatitis is considered to be the ductal hypertension in patients with ductal stones, strictures and stenosis ${ }^{2}$. In general, surgery combined with treatment of pancreatic resection or drainage is done if initial endoscopic treatment is failed ${ }^{3}$. Meanwhile, the distal common bile duct was oppressed by fibrotic pancreas parenchyma secondary to the pancreas fibrosis, which contributed the partial procedures of the CBD stenosis. Surgical treatment may be the only treatment option for chronic pancreatitis with chronic intractable abdominal pain ${ }^{4}$ Specific operations are as follows: resection, decompression (drainage), denervation, and combined surgery. Among all the decompressive or drainage procedures, an analysis of outcome showed that PRP leaded to a substantial quality of life improvement in patients with $\mathrm{CP}^{5}$. PRP combined with cholangioenterostomy is mainly suitable for patients with dilated main pancreatic duct with stones and common bile duct stenosis. At present, individualized treatment was prevalent in the treatment of pancreatitis among the surgeons. Here, we described the result of a 54 years-old male patient with spontaneous chronic pancreatitis with recurrent pancreatic duct and lower common bile duct stenosis, interventional endoscopic treatment failed, after individualized surgical treatment was done successfully.

\section{II.CASE REPORT}

A 54 years-old man was rushed to our hospital complain about intermittent upper abdominal epigastric pain which radiated to back. There was no history of alcohol drinking and no family history of pancreatitis. There was no juvenile chronic pancreatitis in the patient. His past medical history was unremarkable. One of the episodes was gallbladder stones with serious upper abdominal pain with one week of hospitalization, and cholecystectomy was done 8 years back. Common and intrahepatic bile duct were normal, no stones were noticed during the operation and radiographically. Physical examination revealed mild upper abdominal tenderness. Four years ago, the patient was hospitalized again because of recurrent abdominal pain. MRCP and abdominal ultrasound showed lower bile duct stenosis, upper bile duct dilation, stones found in the head of pancreatic duct with the distal dilation, including the removal of pancreatic duct stones and replacement of the stents, contrast examination coated that pancreatic and common bile duct were normal and no obstruction was seen again.

The laboratory test data on admission showed that serum alanine aminotransferase (ALT), aspartate aminotransferase (AST), alkaline phosphatase (ALP), gamma glutamyl transferase (gamma -GT), total bilirubin (TBIL), direct bilirubin (DBIL) were in normal range, Except carbohydrate antigen 199,356.36 U/ml (normal value 0 to $37 \mathrm{U} / \mathrm{ml}$ ), tumor markers, such as car carcinoembryonic antigen, carbohydrate antigen 125,153 and Alpha-fetoprotein (AFP), were within the normal ranges at $1.24 \mathrm{ug} / \mathrm{L}$ (normal value 0 to $13.4 \mathrm{ug} / \mathrm{L}$ ), $5.00 \mathrm{U} / \mathrm{ml}($ normal value 0 to $35.0 \mathrm{U} / \mathrm{ml}$ ), and $6.50 \mathrm{U} / \mathrm{ml}$ (normal value 0 to $31.3 \mathrm{U} / \mathrm{ml}$ ), respectively. Blood / Urine amylase were normal. All common viral markers were (-). Computed tomography (CT) scans showed diffuse enlargement of the pancreas with increased uniformity (Figure 1). Other abnormalities included marked dilation of the body/tail of the pancreas and also upper common bile duct expansion. Earlier endoscopic retrograde cholangiopancreatography (ERCP) performed two times, showing similar results with CT without any malignant tumor observation. Founded on the biochemical sign of cholestasis from the raise of ALP, $\gamma$-GT and TBil, the clinical diagnosis of patient with CT was distal dilation and chronic pancreatitis. Throughout these period of time, steady development of the pancreatic parenchyma lesions were observed in a CT images (Fiture1), with mass in the head of pancreas and tortuous dilated pancreatic duct up to10 $\mathrm{mm}$ were found, presence of calcifications and stones.

Simultaneously, conservational medical could not work and a slow weight loss noted of the patient body. Due to repeatedly recurrence after ERCP and stents replacement, patient whose age was 54 years planned for surgical treatment. Looking to the age of our patient, to avoid additional endoscopic procedures, surgical treatment is preferred. After discussing various treatment options with our patients, the priority was given to the drainage in order to maximize the retention of the pancreatic tissue. Partington-Rochelle binding cholangioenterostomy was chosen as the first choice, in order to better drain the distal pancreas and relieve pain. Considering that CA-199 was slightly high, intraoperative frozen section was essential in partial pancreatic specimen and after rapid intraoperative pathological diagnosis was achieved, the operation was conducted as we had thought smoothly. Follow up was performed weekly for the 1st month after surgery on out-patient bases and then every 3 months. Partington-Rochelle combined with cholangioenterostomy was performed under general anesthesia, without intraoperative and postoperative complications. The main pancreatic duct lengthwise was opened in the area of pancreatic neck, body and tail, lateral Serous - mucous membrane suture pancreatojejunostomy binding the end-to-side cholangioenterostomy and then drained into the loop of jejunumrespectively. The patient was discharged from hospital on $14^{\text {th }}$ day after surgery. Pathological report confirmed diagnosis of chronic pancreatitis of the resection specimen of the partly pancreas. The overall postoperative courses of the patients were uneventful, and their amylase levels remained normal. The patient recovered completely and within 5 weeks returned to work. Three years later, no complications occurred, such as obvious weight loss, jaundice, diabetes mellitus and diarrhea, etc., after a follow-up of 72 months, the patient showed normal liver function, renal function, so that others. At present, the patient in the outpatient follow-up under the conrol stage, the main thing is that he has no sign and symptom of pain. 


\section{III.FIGURES}

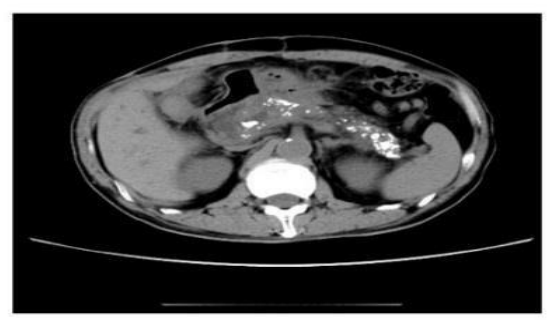

图 1

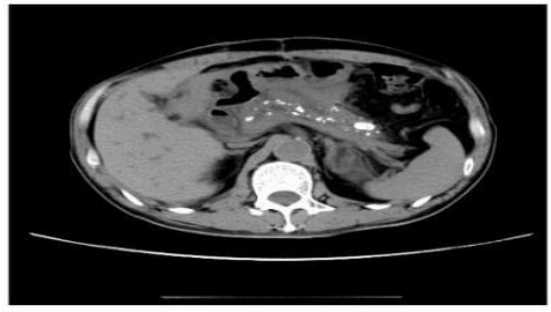

图3

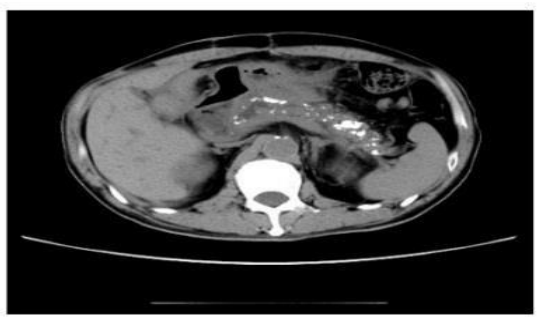

图2

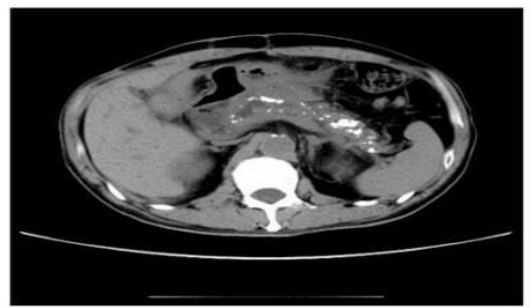

图4

Figure1: Pancreas volume increased significantly in the pancreatic head, and see scattered punctate high-density opacities, dilated pancreatic duct

\section{III.DISCUSSION AND CONCLUSION}

$\mathrm{CP}$ is rather common in our clinics, as well as the Bile duct stenosis, while CP simultaneously accompanied with bile duct stricture is an extremely rare condition, though some article on it were reported. Pancreatic parenchymal fibrosis and ductal hypertension are the main causes of pain in patients with chronic pancreatitis (CP). Two popular hypotheses contribute to the cause of chronic pancreatitis pain: the enlargement of the first sensory nerve diameter in the inflammatory area, indicating the loss of their neural sheath and inflammatory cell infiltration. Pancreatitis specific neuritis has been found to contribute to the clinical pain syndrome by the pain hormone such as substance $\mathrm{P}$ and CGRP local liberation. The 2nd hypothesis recommends that progressive fibrosis leads to enhanced pancreatic duct and tissue pressure, which can easily lead to a pancreatic compartment syndrome due to decreased blood flow ${ }^{6}$. The incidence of common bile duct stricture (CBD) varies widely in chronic pancreatitis $(\mathrm{CP})$. In around $85 \%$ population, the length of the pancreatic segment of the CBD through the pancreatic head and CBD varies from 1.5 to $6 \mathrm{~cm}$, accounting for the variability seen in the narrow length of the clinical practice ${ }^{7}$. CBDS is a recurrent acute inflammatory attack that may eventually lead to fibrosis around the bile duct [10]. This usually occurs in the advanced CP, the highest rate of variation in calcification, anatomy of the common bile duct (CBD) and the head of the pancreas is the narrow nature of influence in the CP. Once the fibrosis of pancreas parenchyma leads to morphological changes of pancreas, the intra-pancreatic part of the CBD may get oppressed inordinately, which contributes to the partial procedures of the CBD stenosis. Since the disease is irreversible, almost all of the treatment efforts are aimed at managing pain and complications with CP. So management aims at relieving pain and preventing further damage ${ }^{8}$. Ever since the first CP patient received the surgical treatment, there have been nearly 50 years. Meanwhile, there are many surgical methods for the treatment of pain in chronic pancreatitis. In 1960, Partington, based on the previous Puestow procedures, come up with the improved Puestow.Improved Puestow side anastomosis has been shown to improve symptoms and accelerate the recovery of normal lifestyle in maintaining low morbidity and mortality. Some critics have said that with the advancement of technology and techniques, endoscopic therapy is to provide a lot of unique advantages with CP treatment, surgery in the first-line management of many patients. In the correct choice of patients, the success rate is high, the incidence is low. The procedure can be repeated with no additional risk, unlike the morbidity and difficulty related with repeat surgery ${ }^{9}$. However, with endotherapy therapy being highly reoccurrence,the best outcome for the debate over surgical treatment is controversial ${ }^{10}$. Tissue and duct hypertension are considered to be the main causes of pain in patients with chronic pancreatitis (CP). Duct dilatation is a result of duct obstruction due to scar or duct stones. However, the technique of choice, drainage or resection is still under discussion. For biliary strictures of $\mathrm{CP}$, surgery has better long-term outcomes compared with endoscopic treatment, furthermore the recurrence rate of the latter is rather high ${ }^{11}$. In addition, the incidence of stent dislocation was significantly greater in cases with stenosis of the common bile duct, especially the distal stent dislocation ${ }^{12}$. Stent dislocation means movement of stent distal end into common bile duct which can cause complications associated with biliary stenting, such as biliary obstruction and cholangitis, etc. The potential risk factors for migration are just secondary to benign disease. Thus, for cases who can't tolerate surgery, insertion of a stent can be a valuable alternative to surgical treatment ${ }^{13}$, or can be used for instant symptom relief and as "bridge to surgery and/or bridge to decision, but are not appropriate for ultimate treatment of CP-related CBD strictures because of long-term poor results ${ }^{14}$. Now it is widely recognized that chronic pancreas is progressive, unrecoverable damage. Choosing the appropriate timing of surgery and striving for early surgery, can reduce the destruction of pancreatic cells, thereby reducing the associated internal and external secretory dysfunction complications. Here we analyze the case operated with Roux-en-Y combined with a Partington-Rochelle duct drainage in cases of chronic pancreatitis with several stenosis and segmental dilatation of the main ducts, also with the stenosis of the common bile 
duct secondary to the pancreatitis. This patient had a badly complicated history which have gone through the stents insertion twice ever. Before any attempt at treatment, malignancy must be excluded ${ }^{14}$. So Rapid intraoperative pathological diagnosis should be made, in order to exclude the possibility of malignancy, which decided to how to choose the surgical procedure. After gastro-colic ligament was well cut and fully opened the lesser omentum, what was exposed to us is that diameter of the common bile duct, about $2.0 \mathrm{~cm}$, which was later confirmed by biopsy, and then removed the original stent of bile duct. Via a T tube, contrast examination confirmed the common bile duct was obstructed, considering that the oppression from the inflammation of the pancreas was the key factor. CT showed that patients was presented with diffuse calcification of the pancreas or branch pancreatic duct stones, pancreatic duct full of stones. So that longitudinally incised the main pancreas, contributed to pancreatic main and branch duct drainage. ERCP images showed the patient's main pancreatic duct dilated not uniformly, and therefore it was rather easier to locate the position of the main pancreatic duct and removed the bracket and a few pieces of stone. So a V line cut of the pancreas was not necessary (Hamburg surgery), which thereby reduced functional pancreatic tissue removed. In addition, the preoperative evaluation and exploration showed the Wirsung (in the head) no apparent stenosis, body and tail pancreatic duct dilatation and no uniform stenosis. So that only the removal of the pancreatic head can't completely relieve the pain, we simply exclude Frey and beager surgery. Considering that the patients had a history of stent implantation with common bile duct stenosis recurrent twice. In order to prevent it from restenosis again, after analyzing the comprehensive preoperative examinations, fast pathological findings and intraoperative exploration analysis, Partington-Rochelle combined with cholangioenterostomy was chosen: $20 \mathrm{~cm}$ from teres ligament, far-end jejunum was cut off $\mathrm{t}$, and by closing device closed the end. Lifted the distal jejunum, and opened the hepato-gastric ligament and side-to-side pancreatojejunostomy was made in the back of the stomach. 10 $\mathrm{cm}$ from pancreatic jejunal anastomosis is for biliary jejunum end-side anastomosis, 3-0 micro-Joe fullthickness continuous suture line. Similarly, $40 \mathrm{~cm}$ below from the biliary intestinal anastomosis was for jejunum side to side anastomosis. Chronic inflammation of the pancreas is hard in texture, so we make pancreatic envelope and jejunal mucosa continuous suture so as to make full use of intestinal mucosal anti-pancreatic characteristics and reduce the probability of the occurrence of pancreatic fistula, concurrently adequate drainage of main pancreatic duct stones and branch pipe in which small stones can't be removed. Also, this kind of surgical approach to maximize retained the pancreatic exocrine function, reduce the probability of postoperative reduction of glucose tolerance, pancreatic dysfunction caused by diarrhea, nutrient absorption disorder, achieved the ideal effect of surgical treatment. As we all know, the principle of individualized treatment for chronic pancreatitis is important, but specific to the choice of surgical approach to each patient should choose what kind of surgery, there is still no a standard rule. For treating this sort of complicated chronic pancreatitis, the ESGE recommends surgical drainage as a first-line therapy. For treatment of chronic pancreatitis associated with biliary strictures, the high-quality option between endoscopic and surgical therapy should rely on local expertise, patient comorbidities and expected patient compliance with repeat endoscopic procedures ${ }^{15}$. Therefore, we will make our efforts for the future realization of standardized rules

\section{Acknowledgements}

This funding for the completion of this case report was kindly provided by Jiangsu Planned Projects for Postdoctoral Research Funds, No.1302096B; and the Zhenjiang Science and Technology Committee (No.SH2014089). All authors have reviewed and confirmed, furthermore, there is no any conflict of interest

\section{REFERENCES}

[1].Etemad B, Whitcomb DC. Chronic pancreatitis: diagnosis, classification, and new genetic developments. Gastroenterology. Feb 2001;120(3):682-707.

[2].Strobel O, Buchler MW, Werner J. Surgical therapy of chronic pancreatitis: indications, techniques and results. International journal of surgery. Aug 2009;7(4):305-312.

[3].Skorzewska M, Romanowicz T, Mielko J, et al. Frey operation for chronic pancreatitis associated with pancreas divisum: case report and review of the literature. Przeglad gastroenterologiczny. 2014;9(3):175-178.

[4].Giulianotti P, Gorodner V, Kinzer K, Benedetti E, Oberholzer J. Robot-assisted pancreatoduodenectomy with preservation of the vascular supply for autologous islet cell isolation and transplantation: a case report. Journal of medical case reports. 2012;6(1):74.

[5].Pakosz-Golanowska M, Post M, Lubikowski J, et al. Partington-rochelle pancreaticojejunostomy for chronic pancreatitis: analysis of outcome including quality of life. Hepato-gastroenterology. Sep-Oct 2009;56(94-95):15331537.

[6].Schlosser W, Schwarz A, Beger HG. Surgical treatment of chronic pancreatitis with pancreatic main duct dilatation: long term results after head resection and duct drainage. HPB : the official journal of the International Hepato Pancreato Biliary Association. 2005;7(2):114-119.

[7].Abdallah AA, Krige JE, Bornman PC. Biliary tract obstruction in chronic pancreatitis. HPB : the official journal of the International Hepato Pancreato Biliary Association. 2007;9(6):421-428.

[8].Jha AA, Kumar M, Galagali A. Management options in chronic pancreatitis. Medical journal, Armed Forces India. Jul 2012;68(3):284-287.

[9].Tandan M, Nageshwar Reddy D. Endotherapy in chronic pancreatitis. World journal of gastroenterology. Oct 07 2013;19(37):6156-6164.

[10]. Ceppa EP, Pappas TN. Modified puestow lateral pancreaticojejunostomy. Journal of gastrointestinal surgery : official journal of the Society for Surgery of the Alimentary Tract. May 2009;13(5):1004-1008. 
[11]. Regimbeau JM, Fuks D, Bartoli E, et al. A comparative study of surgery and endoscopy for the treatment of bile duct stricture in patients with chronic pancreatitis. Surgical endoscopy. Oct 2012;26(10):2902-2908.

[12]. Kawaguchi Y, Ogawa M, Kawashima Y, et al. Risk factors for proximal migration of biliary tube stents. World journal of gastroenterology: WJG. Feb 7 2014;20(5):13181324.

[13]. Waldthaler A, Schutte K, Weigt J, Kropf S, Malfertheiner P, Kahl S. Long-term outcome of self-expandable metal stents for biliary obstruction in chronic pancreatitis. JOP: Journal of the pancreas. Jan 2013;14(1):57-62.

[14]. Familiari P, Boskoski I, Bove V, Costamagna G. ERCP for biliary strictures associated with chronic pancreatitis. Gastrointestinal endoscopy clinics of North America. Oct 2013;23(4):833-845.

[15]. Dumonceau JM, Delhaye M, Tringali A, et al. Endoscopic treatment of chronic pancreatitis: European Society of Gastrointestinal Endoscopy (ESGE) Clinical Guideline. Endoscopy. Aug 2012;44(8):784-800. 